\title{
What Hamblin's Formal Dialectic Tells About the Medieval Logical Disputation ${ }^{1}$
}

\author{
Pavlova Alexandra Mikhailovna \\ Institute of Philosophy, Saint Petersburg State University \\ 5 Mendeleevskaya Liniya, St. Petersburg, 199034, Russian Federation \\ E-mail: alexandra22@mail.ru
}

In this paper we reconstruct a famous Severin Boethius's reasoning according to the idea of the medieval obligationes disputation mainly focusing on the formalizations proposed by Ch. Hamblin. We use two different formalizations of the disputation: first with the help of Ch. Hamblin's approach specially designed to formalize such logical debates; second, on the basis of his formal dialectics. The two formalizations are used to analyze the logical properties of the rules of the medieval logical disputation and that of their formal dialectic's counterparts. Our aim is to to show that Hamblin's formal dialectic is a communicative protocol for rational agents whose structural rules may differ, thus, varying its normative character. By means of comparing Hamblin's reconstructions with the one proposed by C. Dutilh-Novaes we are able to justify the following conclusions: (1) the formalization suggested by Hamblin fails to reconstruct the full picture of the disputation because it lacks in some the details of it; (2) Hamblin's formal dialectic and the medieval logical disputation are based on different logical theories; (3) medieval logical disputation, represented by the formalization of C. Dutilh-Novaes, and the two ones of Hamblin encode different types of cognitive agents.

Keywords: formal dialectics, game, medieval disputes of obligationes, dialogue logic, argumentation, Hamblin, belief revision

\section{Introduction}

The study of the medieval logical disputation lies in between two different areas of research, namely: the history of logic and some modern trends in logic used for the reconstruction of the disputation. One of

\footnotetext{
${ }^{1}$ The research is supported by the Russian Foundation for Humanities, project № 14-03-00650.
}

(C) Pavlova A.M. 
these trends is associated with the development of the logical game theory. A weighty contribution to them both was made by an Australian logician Charles Hamblin who tried to model a medieval logical disputation with the help of a specially modified version of his own logical system called 'the formal dialectic'. He describes his system, first presented in the book Fallacies, published in 1970, as follows:

Dialectic, whether descriptive or formal, is a more general study than Logic; in the sense that Logic can be conceived as a set of dialectical conventions. It is an ideal of certain kinds of discussion that the rules of Logic should be observed by all participants, and that certain logical goals should be part of the general goal [9].

Among the distinguishing features of the dialectical disputation that makes it different from the formal deduction one should mention the number of participants, namely more than one, and the existence of a functional communication protocol for those participants ${ }^{2}$ that can be presented in the game-theoretical form [13]. In chapter 8, called 'Formal Dialectic', of the Fallacies, Ch. Hamblin considers the disputations de obligationibus, or simply obligationes, as obligation games. On the basis of the proposed system he gives formalisation of the two main types of disputations de obligationibus, namely: the standard one, described by Burley, and the alternative one of William of Sherwood.

Although, the obligation game is claimed to represent a formalization of the medieval disputation de obligationibus, there still remains an issue with respect to its adequacy. In the present paper we draw a comparison between Ch. Hamblin's disputations along with his system of formal dialectic to the medieval disputations de obligationibus as they are described in contemporary literature. We take a modern reconstruction of that medieval disputation for the comparison because formal systems can be correctly compared to formal systems only, but not to informal conceptions, once formal criteria are used, such as truth values, inference rules etc. We can formulate our subject matter in a form of a question whether the obligation games, as a particular case of the system of formal dialectic, can be considered a satisfactory and

\footnotetext{
${ }^{2}$ Sometimes those participants are called 'agents'.
} 
adequate formalization of the medieval disputation de obligationibus; or whether it lacks or corrupts some of the essential features of the medieval disputation.

However, being formulated as is shown above this problem would be of interest merely for the historians of logic. That is why it is necessary to clarify the incentive that underlies the analysis to be presented in this article. The paper's objective is to show that Hamblin's formal dialectic is a communicative protocol for rational agents whose structural rules may differ, thus, varying its normative character. For instance, in the paper we use classical propositional logic as structural rules following [9]. Furthermore, obligation game proposed by Hamblin to model medieval disputation is merely a restricted variant of formal dialectic which we claim to be a sort of cognitive game. We try to justify here the claim that formal dialectic is a more general theory than logic incorporated by the players. In the cours of the paper we reveal some features of both formal dialectic and medieval disputation de obligationibus. This requires the analysis of the roles that cognitive agents play in the systems in question. We use three main criteria: ${ }^{3}$ that we use to explain some differences between formal dialectic and medieval disputations: (1) epistemic, (2) deductive and (3) goal-oriented (or actional and dynamic) [15]. Truth can represent both epistemic and deductive aspects of agents. As an epistemic criterion it shows what agents can know or/and believe in, which is related to some semantics. However, a semantic model corresponds to some specific set of inference rules that agents may use, and thus, it is related to deductive parameters. As far as rules of interaction are concerned, they influence the type of a system that a dialogue or a dispute represents [19], as it determine the formulae that might be inferred in its course. Thus, they can be associated with deductive competences of agents. At last, additional terms and conditions might partially take the shape of agents' goals and intentions so we associate those conditions with action (goal-oriented) elements of a cognitive agent.

\footnotetext{
${ }^{3}$ We we use the term 'intellectual competences' of agents to refer to those criteria.
} 
This will allow us to show how the difference in those basic principles that we shall find in the course of our comparison between the medieval disputation de obligationibus and its formalization presented by Ch. Hamblin together with his own system of formal dialectic can effect those basic notions. At the same time we shall see whether those systems encode one and the same type of agent or different ones. By the type of agents we understand differences in their cognitive presumptions influencing the reasoning and actions. In the paper, we claim that the formal dialectic and medieval disputations presuppose different types of agents.

With the general aim formulated we can identify some intermediate tasks, which accordingly define the structure of the present paper, as follows:

1) give a brief overview of the main concepts and rules of the medieval disputations de obligationibus and their formal representation;

2) reconsider and discuss the rules for Hamblin's obligation games which is presented by Ch. Hamblin as a formalization of the medieval disputation;

3) have a look at the basic elements of Hamblin's system of formal dialectic from a general perspective;

4) and finally, compare Hamblin's system of formal dialectic to the medieval disputations de obligationibus.

To illustrate our analysis we shall discuss an example based on the treatise De Hebdomadibus by Boethius [2]. For the simplicity we leave out some details and take only the following argumentation:

Things which exist are good. For the common view of the learned holds that everything which exists tends toward good. But everything tends toward its like. Therefore, the things which tend toward good are themselves good.

But we have to ask how they are good, by participation or by substance? If by participation, they are in no way good in themselves. For what is white by participation is not white in itself in virtue of the fact that it itself 
has being. And the same applies to other qualities. Therefore, if they are good by participation, they are in no way good in themselves. Therefore, they do not tend toward good. But that was granted. Therefore, they are not good by participation but by substance.

With the goals set, it is necessary to specify the following terms:

- by disputation de obligationibus we understand a particular kind of medieval logical disputation represented in treatises of Walter Burley, Richard Kilvington and Roger Swynesed and which is discussed in section 1 ;

- medieval logical disputation, i.e.,a general term used for the genre of medieval disputations;

- obligation game, i.e., the system specificly proposed by Ch. Hamblin to formalise the disputation de obligationibus;

- the system of formal dialectic (or simply formal dialectic), i.e., a system of regulated dialogues or family of dialogues with 'at least two participants who speak in turn in accordance with a set of rules or conventions' [9, p. 255] that is aimed at exceeding 'the bounds of Formal Logic; to include features of dialectical context within which arguments are put forward [9, p. 254].

\section{What is Disputation de Obligationibus?}

In this section we give an overview of the disputations de obligationibus and their history. First of all, it is believed that the scholastic theories on obligations were inspired by Aristotle's Topics and undergone some changes in the $X I V^{t h}$ century. One should distinguish the theory proposed by Walter Burley known as antiqua responsio and those of Richard Kilvington and Roger Swyneshed known as nova responsio. The latter treat obligations in epistemic terms which makes, as C. Dutilh-Novaes suggests in [6], it possible to consider those disputations as a theory of belief revision or counterfactuals. In this article we shall concentrate on the obligationes theory of Burley [4], [22].

The disputation de obligationibus is a kind of medieval disputation with two participants: Opponent and Respondent. In our description 
of the disputations we shall follow works of C. Dutilh-Novaes [6] and E.Lisanyuk [14]. According to the way the Respondent should evaluate the thesis of the disputation, one can distinguish between several types of obligations like positio, depositio, dubitatio, impositio, petitio and others. However, we are going to consider only one and the most widespread types called positio. It consists of positum, propositum, the phrase 'cedat tempus' indicating the end of the disputations and victory of the respondent. There might be two optional elements: casus and petitio as well. Those elements are described as follows:

a) Positum is the basic element of the disputation that serves it as a thesis that the Respondent either accepts and then the disputation starts, or denies it and then the dispute fails to begin. Sometimes there are two more elements added to the positum, i.e., casus and petitio representing special conditions and constraints that together with positum form what is called positio, i.e., the whole set of thesis propositions;

b) Propositum represents a sentence put forward by the Opponent for the Respondent to evaluate and either accept or deny given the positio.

c) There exists a set of rules for time reading, including the phrase 'cedat tempus' meaning the time is over.

d) A set of agents, or players, consisting of two players with asymmetric roles: Opponent and Respondent.

The opponent puts forward some proposition or a set of propositions called positum and positio respectively. Positio represents a thesis of the disputation. The respondent is supposed to evaluate it as:

i) possibly true,

ii) possibly false or

iii) a proposition with unknown logical value. 
We shall consider only the first case in which the thesis was evaluated as possibly true. On the basis of the evaluation the respondent can admit the positum or deny it. After the evaluation is provided and the respondent has admitted the thesis the disputation starts.

There are two types of acts in the dialogue, which are presented by two roles: that of the opponent and that of the respondent. The role of the opponent consists in asking questions whereas the respondent is forced to answer. This shows us that the roles in the disputation de obligationibus are asymmetric. One can easily see that opponent is unforced as he himself chooses which proposition to bring up and so he also chooses a number of strategies for the respondent from the whole set of possible strategies whereas respondent is, firstly, obliged to give an answer and evaluate the proposition put forward and, secondly, she does not choose independently the strategy, but has to select one of the strategies previously picked out by the opponent.

The respondent may also be suggested to evaluate additional information contained in the proposals presented in the form of casus and petitio. Casus, or actual fact, and petitio represent the additional description of state of affairs. State of affairs is an important part of the disputation as it serves as a correlate of the evaluation of the propositions. The propositions are sentences brought up by the opponent in any step after the positum has been accepted. The respondent has to evaluate such propositions, having found out whether they are relevant to the positum and proposita, conceded in the earlier steps, according to the rules specified below. We shall distinguish between steps and rounds. By a step we understand each move of a player, whereas a round is formed by a question of the opponent and the respondent's answer. Rounds can be open or closed. We shall call some round open if and only if it has a question by the opponent, but not an answer of the respondent. Otherwise, the round is closed. It is easy to see that an open round is formed by a single step only, whereas a closed round has to contain two steps. For each step $n$ of the disputation, beginning with the first propositum, the propositum is 'sequentially relevant' at step $n$ if and only if it satisfies the following conditions: 
(1) it logically follows from the conjunction of the positum together with any proposita that have been conceded at earlier steps of the disputation;

(2) it logically follows from the conjunction of the positum together with the contradictories of any proposita that have been denied at earlier steps.

It is 'irrelevant' at step $n$ if and only if it is neither sequentially relevant nor incompatibly relevant there. On the basis of relevance of the propositum to the earlier conceded obligations the respondent evaluates the propositum. Thus, each proposition accepted at any point $n$ of the disputation (starting from the thesis) becomes an obligation (obligatio) of the Respondent which serves as a foundation for the further evaluation of propositions.

For each step $n$ of the disputation, and for each propositum $p, p$ is true if it is sequentially relevant at $n$, and false if it is incompatibly relevant at $n$. After that, if the propositum was evaluated as true, the respondent has to concede it, if false, she has to deny it. If $p$ is irrelevant in step $n$, the respondent has to evaluate it according to her knowledge of the actual facts. Thus, if $p$ is irrelevant at $n$ and the respondent knows it is true in fact, she should concede it; if $p$ is irrelevant at $n$ and the respondent knows it is false in fact, she should deny it; if $p$ is irrelevant and the respondent does not know whether it is true or false, she should doubt it.

\section{Obligation Games as a Formalisation of the Disputation de obligationibus}

In this section we present the formalization of the medieval disputation proposed by Charles Hamblin which he called 'Obligation game'. First of all he specifies the language of the game as 'a finite propositional language based on elementary propositions' $a_{0}, a_{1}, \ldots, a_{n}$ and 'truth-functional operators, supplemented with several special locutions' [9, p. 260]. However, let us note that 'in place of propositional calculus we could substitute any other finite language of sufficiently normal type, for example, lower predicate calculus on a universe with 
finitely many individuals and limited variety' [9, p. 260]. Neither did Ch. Hamblin formalize the rule-language, nor did he care for historical accuracy and details as he used this type of disputation to illustrate his own system of formal dialectic and the origin of some fallacies. Thus it differs a bit from the other formalizations of the obligations, like those presented in the works of C. Dutilh-Novaes and E. Lisanyuk.

Let the disputation be specified by the tuple $\langle\Pi, O, P, F, W, C\rangle$, where:

- $\Pi$ stands for the set of players. Obligation game normally has two players. Those players are given one of the two possible roles: the role of the opponent or the one of the respondent.

- $O$ is an ordered set of propositions, put forward by the opponent to the respondent for the evaluation.

- $O=\left\{o_{0}, o_{1}, \ldots, o_{n}\right\}, n \geq 0$, where $n$ is a position of the proposition in the obligation game, starting from $o_{0}$ which stands for the thesis.

- $P$ is an ordered set of propositions, consisting of the answers of the respondent: $P=\left\{p_{0}, p_{1}, \ldots, p_{n}\right\}, n \geq 0$, where $n$ is a position of the proposition in the obligation game, starting from $p_{0}$ which stands for the evaluation of the thesis.

- $P_{j+1}=\left\{p_{0}, p_{1}, \ldots, p_{j-1}, p_{j}, p_{j+1}\right\}$ represents a set of the propositions evaluated by the respondent in the step $j+1$.

- $F$ is the function of evaluation from the set $O$ to the set of logical values $\{1,0\}$, where 1 stands for true, 0 stands for false.

In Hamblin's Obligation game there are only two logical values whereas in other formalizations [6] there occur three logical values $\{1,0, ?\}$, where "?" stand for an indeterminate value. In medieval disputations it seems to be used for the propositions evaluated as irrelevant, and not for the relevant ones. Historicly, the Respondent had a possibility to consider a proposition as doubtful ${ }^{4}$ so the formalisation proposed by Ch. Hamblin

\footnotetext{
${ }^{4}$ if it was irrelevant and neither it nor its negation followed from his or her background knowledge.
} 
does not fully represent the Medieval disputations de obligationibus. The third value is preserved in the systemes proposed by C. Dutilh-Novaes and H. Lagerlund and E.J. Olsson as well.

In his Obligation game Hamblin does not specify the procedure of evaluation and the basis on which the respondent decides whether to concede the propositum or its negation. It can be explained by the supposition that he assumes employing some basic formal system on the lower level of the game, and that the evaluation should proceed with the help of it. However, the same question arises with respect to the formalisation of C. Dutilh-Novaes, as S. Uckelman notices in [23]. In order to keep track of the propositions accepted by the respondent $\mathrm{Ch}$. Hamblin introduces a notion of commitment store. $C$ is a commitment store of the respondent, so that:

- $C_{n}=\left\{p_{0}, p_{1}, \ldots, p_{n}\right\}$, where $n \geq 0, p_{0}$ is positum.

- $C_{j}=C_{j-1} \cup p_{j}$, for each $j=0,1, \ldots, m+1$.

Thus the commitment store is a set consisted of the propositions accepted by the Respondent and the negations of the propositions denied by her. Ch. Hamblin uses the notion of the commitment store for the respondent to specify the requirement of correctness of the respondent's answers. By the correctness of answers we understand here that the answers of the respondent should not form an implicit contradiction with any preposition in the set of previously accepted prepositions. By the explicit contradiction we mean here the existence of two propositios $p_{n}$ and $p_{m}$ such that $p_{n}=a_{i}$ and $p_{m}=\neg a_{i}$.

Surprisingly there is no set $K$ in Ch. Hamblin's Obligation game. In the formalisation of C. Dutilh-Novaes $K$ is an open set of true, false and obscure propositions $k_{0}, k_{1}, \ldots, k_{n},(n \geq 0)$ that form a common knowledge, shared by all the participants of the disputation. Thus, it is not quite clear what basis should the respondent concede or deny the irrelevant propositions on, as if she has to instantiate them. However, as we shall see later from the correctness-rule, Casus might serve as a set specifying the truth value of some irrelevant propositions. Nevertheless, it does not usually contain all possible propositions of the language. 
The Opponent moves first and her first locution has three parts: (a) 'actual fact', (b) 'positum' and (c) 'propositum', as C. Hamblin calls them. They are specified as follows:

(a) Casus, which in Hamblin's system is an obligatory part of the obligation game, consists of the words 'Actual fact' and statement ${ }^{\prime} B^{\prime}$. ' $B^{\prime}$ contains evaluation of the language, consisting of a statedescription $b_{1}, b_{2}, \ldots, b_{m}$, where each $b_{i}=a_{i}$ or $b_{i}=\neg a_{i}$.

(b) Positum consists of word 'Positum', followed by a contingent statement ${ }^{\prime} C^{\prime}$, where ${ }^{\prime} C^{\prime}=o_{0}$.

(c) Propositum consists of the word 'Propositum I' and statement $o_{1}$.

As one could notice, in Hamblin's obligation game (a), (b) and (c) form one single step and, thus, open only one round. This mans that the respondent can either accept (a), (b) and (c) all together or deny all os them ${ }^{5}$. However, in the disputation de obligationibus and its formalization by C. Dutilh-Novaes (b) and (c) represent separate steps and thus open two different rounds. That entails that the respondent has a larger set of strategies to choose: (1) he can accept both (b) and $(\mathrm{c})^{6} ;(2)$ he can deny (b) and then the game does not $\operatorname{start}^{7} ;(3)$ he can accept (b) but deny (c). The strategy number (3) cannot be played out in the Obligation games. We can conclude that in Obligation games the Respondent has less strategies that he can follow according to the structural rules that results in the number of the games he can possibly win. Thus we can make an interim conclusion that the truth in Obligation games is different from the one in the disputations de obligationibus (as well as their formalization by C. Dutilh-Novaes), as the Respondent has less games he is able to win. If we understand casus and propositum as a sort of model specification, the above shows us that in Obligation games the agents start with more precise models than in

\footnotetext{
${ }^{5}$ However, in the latter case the game does not start.

${ }^{6}$ This strategy is equal to the one in he Obligation games where the respondent accepts (a), (b) and (c).

${ }^{7}$ This strategy equals the one in the Obligation games where the respondent denies (a), (b) and (c).
} 
the formalization by C. Dutilh-Novaes. The fact that two rounds at the beginning of the disputations de obligationibus are combined into one round might entrap the Respondent to accept a contradiction. Let us consider the structural rules for an obligation game:

- Answer-rule: Each of the respondent's contributions $p_{n}=o_{n} \mathrm{~V}$ $\neg o_{n}, n \geq 1$.

- Ending-rule: The disputation ends if and only if:

(1) $P_{n}=\perp$; or

(2) $P_{n}=\top$ and cedat tempus, which means 'the time is out'.

\section{- Winning-rule:}

(1) Opponent wins if and only if $P_{n} \models \perp$

(2) Respondent wins if and only if $P_{n} \models \top$ and cedat tempus.

Ch. Hamblin defines the notion of cedat tempus by specifying the number of steps in the game so that the respondent wins if and only if $P_{n}=\top$ and $n=11$.

- Correctness-rule: The respondent's answer $p_{n}$ is correct if and only if it is either

1. implied by $C_{n-1}$, or

2. consistent with $C_{n-1}$ and implied by $B$; otherwise it is incorrect.

The correctness rule shows that Ch. Hamblin does not use the notion of relevance of the propositum to the positum or previously conceded proposita $^{8}$. Thus, there is no difference between sequently relevant and sequently irrelevant propositions because no matter if it is relevant or not the respondent is only obliged to choose whether to concede the

\footnotetext{
${ }^{8}$ We shall add this notion to our reconstruction for the sake of clarity as we do with the concept of common knowledge. Otherwise, the respondent would have no rule according to which she should evaluate propositions.
} 
proposition or its negation, but there is nothing said of his duty to concede sequentially relevant and deny incompatibly relevant. Although, the propositions stated as the actual fact $\left({ }^{\prime} B^{\prime}\right)$ are important as they serve as the correlate of evaluation of respondent's answers as correct or incorrect which was specified in the correctness-rule. Ch. Hamblin's interpretation gives us no hint of the way the Respondent should answer and in that sense she is in no way determined. This still might be partly related to a possible interpretation for the Respondent and propositions relevant to $K_{C}$, thought there are differences with respect to the positum. It is not specified how the Respondent is fined for answering incorrectly, and, as there is no rule to make the Respondent accept formulae deducible from the positum and proposita ${ }^{9}$, there is actually no way to force her to accept a contradiction. However, we shall consider the correctness rule as a forcing one in the example below.

Let us consider an example of the Obligation game, based on the treatise by Boethius [2]. Though the original text is written in a form of a monologue it has such a form that allows for a simple transformation into the dialogue, or better, a logical game.

\footnotetext{
${ }^{9}$ We do not use the notion of commitment store here as it also contains casus which we can view as a $K_{C}$ set for the Obligation game. Though, we might add casus to a system with a common state of knowledge $K_{C}$, but then we should treat it as other proposita except for the fact that it is accepted with the positum as a set of preconditions.
} 


\begin{tabular}{|c|c|c|c|}
\hline & Opponent & Respondent & $\begin{array}{l}\text { Commitment } \\
\text { Store }\end{array}$ \\
\hline 1 & $\begin{array}{l}\text { 1. 'Actual fact': } \\
b_{1} \text { : Everything that is } \\
\text { tends to the good; } \\
b_{2} \text { : Everything tends } \\
\text { towards its like; } \\
b_{3} \text { : That all things that } \\
\text { are, are God is abhorrent; } \\
b_{4} \text { : The things that are, } \\
\text { are good. } \\
2 . \text { 'Positum': } \\
o_{0} \text { : The things that are, } \\
\text { are good by participation. } \\
\text { 3. 'Propositum I': } \\
o_{1} \text { : The things that are, } \\
\text { tend to the good. }\end{array}$ & $\begin{array}{l}p_{1} \text { : I concede that } \\
\text { "The things that are, } \\
\text { tend to be good". } \\
p_{1}=o_{1}\end{array}$ & $\begin{array}{l}C_{1}=\left\{b_{1}, b_{2}, b_{3},\right. \\
\left.b_{4}, o_{0}, o_{1}\right\}\end{array}$ \\
\hline 2 & $\begin{array}{l}\text { 'Propositum II': } \\
o_{2} \text { : The things that are, } \\
\text { are themselves good per se. }\end{array}$ & $\begin{array}{l}p_{2}: \text { I deny that } \\
\text { "The things that are, } \\
\text { are themselves good } \\
\text { per se". } \\
p_{2}=\neg \mathrm{O}_{2}\end{array}$ & $\begin{array}{l}C_{2}=C_{1} \cup\left\{\neg o_{2}\right\} \\
C_{2}=\left\{b_{1}, b_{2}, b_{3}\right. \\
\left.b_{4}, o_{0}, o_{1}, \neg o_{2}\right\}\end{array}$ \\
\hline 3 & $\begin{array}{l}\text { 'Propositum III': } \\
o_{3}: \text { Everything tends } \\
\text { towards its like. }\end{array}$ & $\begin{array}{l}p_{3}: \text { I concede that } \\
\text { "Everything tends } \\
\text { towards its like". } \\
p_{3}=o_{3}\end{array}$ & $\begin{array}{l}C_{3}=C_{2} \cup\left\{o_{3}\right\} \\
C_{3}=\left\{b_{1}, b_{2}, b_{3}\right. \\
\left.b_{4}, o_{0}, o_{1}, \neg o_{2}, o_{3}\right\}\end{array}$ \\
\hline 4 & $\begin{array}{l}\text { 'Propositum IV': } \\
o_{4} \text { : The things that are, } \\
\text { do tend to the good. }\end{array}$ & $\begin{array}{l}p_{4}: \text { I deny that } \\
\text { "The things that are, } \\
\text { do tend to the good". } \\
p_{4}=\neg O_{4}\end{array}$ & $\begin{array}{l}C_{4}=C_{3} \cup\left\{\neg o_{4}\right\} ; \\
C_{4}=\left\{b_{1}, b_{2}, b_{3},\right. \\
b_{4}, o_{0}, o_{1}, \neg O_{2}, o_{3}, \\
\left.\neg o_{4}\right\}\end{array}$ \\
\hline 5 & 'Win and Finish' & & \\
\hline
\end{tabular}

In order to explicate the run of the obligation, and the Respondent's answers in particular, we need to add some comments with respect to each round as the game proceeds. Starting from the first round, it is clear that $b_{1} \models o_{1}$. The respondent admits the positum and then can choose whether to concede or deny the propositum I. As the propositum I is irrelevant to the positum the respondent can evaluate this proposition according to the common knowledge. However, it is relevant to the 
special conditions of the game, or actual fact (casus), the respondent should concede the propositum I in order to answer correctly.

In the round 2 one can notice that $o_{0}=\neg O_{2}$. "For what is white by participation is not white per se in that it is, and the same in the case of other qualities. If they are good by participation, then, they aren't themselves good per se in any way". That means that if something is good by participation, it is neither good by substance, nor by itself (per se). Propositum II is relevant to the positum. Thus, it follows from the positum that propositum II is false and the respondent must deny it.

As for the round 3, the Respondent concedes the proposition because of $b_{2}=o_{3}$. Although, the propositum III is irrelevant to the positum (though Hamblin does not use the conception of relevance) it is relevant to the special conditions of the game, or actual fact (casus), the respondent should concede the propositum III in order to answer correctly.

Finally, in round 4 the Respondent uses $o_{3} \wedge \neg O_{2} \models \neg O_{4}$ to deny the proposition. The opponent repeats once more the question $o_{1}$, but now it is relevant and according to the rules of the game the respondent has to deny the proposition.

This example shows us a possible flow of the obligation game as a reconstruction of the disputations de obligationibus. The opponent finishes the game and she has won because the respondent's last answer $\left(p_{4}\right)$ was incorrect as her commitment store $\left(C_{4}\right)$ became inconsistent as $p_{4}$ and $p_{1}$ together make a contradiction. This happens because $p_{1}=o_{1}$ and $p_{4}=\neg o_{4}$, where $o_{1}=o_{4}$, so that we can restate the respondent's commitment store as follows: $C_{4}=\left\{b_{1}, b_{2}, b_{3}, b_{4}, o_{0}, o_{1}, \neg o_{2}, o_{3}, \neg o_{1}\right\}$.

Actually, as there are not any notions of sequently relevant and sequently irrelevant propositions, the respondent could accept the proposition $o_{4}$ : "The things that are, do tend to the good" in the round number 4 of the previous example, but we still would have end up with a contradiction in the commitment store $C_{4}$, because it already existed in the step number 3 in the commitment store $C_{3}$, though it was implied, i.e., needed some logical inference from the propositions in $C_{3}$ for its explication. However, we should emphasize that Ch. Hamblin has not specified 
any inference rules for his system, so we should assume that along with accenting a language of some logical system we should use its inference rules and axioms as well.

Nevertheless, we suppose that Hamblin writes nothing about those rules as it is not particularly important as his aim is to show how his structural rules can influence the system. We shall face this problem once again while considering his system of formal dialectic in the next section 3 .

\section{The Difference between the Obligation Games and Formal Dialectic}

In the previous section 2 we have considered the Obligation game which is supposed to be a formalization of the disputations de obligationibus. We have specified some of the features of those games as well. Now we shall briefly present the system of formal dialectic and see how we may use it to formalize disputations. So, similar to the obligation game, Hamblin does not pay much attention to the rules of inference according to which the players can make their moves which could mean that any set of rules making valid inference can work. Thus, different players can use different sets of rules with still good results in the game. Neither does he explicitly specify any logical model with for the game. However, we suppose that one can take some logical system and see how those rules of the game affect the number of true formulae. Therein after we shall specify some rules for the formal dialectic together with providing a general characteristics for it.

However, we would like to start with considering the same example as in the previous section but formalized using the formal dialectic. To simplify our representation we shall use the following notation:

- $p:=$ The things that are, tend toward good;

- $q:=$ The things that are, are themselves good per se;

- $r:=$ Everything tends towards its like. 


\begin{tabular}{|l|l|l|l|l|}
\hline & $\begin{array}{l}\text { White's } \\
\text { Commit- } \\
\text { ment Store }\end{array}$ & White & Black & $\begin{array}{l}\text { Black's } \\
\text { Commit- } \\
\text { ment Store }\end{array}$ \\
\hline 1 & $p$ & $?(p)$ & $\operatorname{Com}(p)$ & $p$ \\
\hline 2 & $p, q$ & $?(q)$ & $\operatorname{Com}(\neg q)$ & $p, \neg q$ \\
\hline 3 & $p, q, r$ & $?(r)$ & $\operatorname{Com}(r)$ & $p, \neg q, r$ \\
\hline 4 & $\begin{array}{l}p, q, r, \\
(\neg q \wedge r) \rightarrow \neg p\end{array}$ & $?((\neg q \wedge r) \rightarrow \neg p)$ & $\operatorname{Com}((\neg q \wedge r) \rightarrow \neg p)$ & $\begin{array}{l}p, \neg q, r, \\
(\neg q \wedge r) \rightarrow \neg p\end{array}$ \\
\hline 5 & $\begin{array}{l}p, q, r, \\
(\neg q \wedge r) \rightarrow \neg p, \\
p \vee \neg p\end{array}$ & $?(p \vee \neg p)$ & $\operatorname{Com}(\neg p)$ & $\begin{array}{l}\neg p, p, \neg q, r, \\
(\neg q \wedge r) \rightarrow \neg p\end{array}$ \\
\hline 6 & $\begin{array}{l}p, q, r, \\
(\neg q \wedge r) \rightarrow \neg p\end{array}$ & $\operatorname{Resolve}(p \vee \neg p)$ & $\operatorname{No}(p)$ & $\begin{array}{l}\neg p, \neg q, r, \\
(\neg q \wedge r) \rightarrow \neg p\end{array}$ \\
\hline
\end{tabular}

Now we shall specify the rules used in the above example. First of all we can divide all the rules of a dialectical system into two groups:

- Syntactic rules that govern the way the players act in the dialogue providing possible moves

- Rules that determine operations over commitment store determining what propositions are inserted or deleted from the commitment store.

Definition 1. We can define the language of dialectical system $D=$ $\langle$ Prop, Const, $\Phi\rangle$ as follows:

1. Propositional variables $\operatorname{Prop}=\left\{p, q, r, p_{1}, q_{1}, r_{1}, p_{2}, q_{2}, r_{2}, \ldots\right\}$;

2. Standard propositional constants Const $=\{\vee, \neg, \rightarrow, \wedge\}$. Ch. Hamblin does not define rules for conjunction, however, he uses it in one of the examples. So we tried to extract the rule from the example of the dialogue [9, p. 267];

3. Functors for dialectical actions $\Phi=\{$ Com, No, ?,Why, Resolve $\}$ defined as follows: 
(a) $\operatorname{Com}(A)$ is an 'utterance $A$ ', sometimes it is possible to use $\operatorname{Com}(A, B)$. The uttered formula is added to the commitment store of both a speaker and a hearer with some exceptions.

(b) $N o(A, B, \ldots, C)$ stands for 'no commitment $A, B, \ldots, C$ ' and deletes any $A, B, \ldots, C$ from the commitment store of the speaker.

(c) ? $(A, B, \ldots, C)$ is a 'question $A, B, \ldots, C$ ', $n \geqslant 1$, where $n$ is a number of propositions. We suppose that comma stands for disjunction here, i.e. ?( $A \vee B \vee \ldots \vee C)$. This functor inserts the disjunction $A \vee B \vee \ldots \vee C$ into the commitment stores of both speaker and hearer with some exceptions;

(d) Why $(A)$, for any proposition $A$ if it is not an axiom. It is a request for argumentation;

(e) $\operatorname{Resolve}(A)$ is a request for resolution. It does not influence any commitment store.

REMARK 1. The status of the resolutions in the formal dialectic is not quite clear, however, we can give a few interpretations of its function:

- We can view resolution as a request given to the other participant to identify whether formula $A$ or $\neg A$ is consistent with his commitment store. As a result the other participant should answer either $N o A$ or $N o \neg A$. Thus we might consider resolution to be a sort of consistency test.

- It is also possible to suppose that resolution is a sort of belief revision operator. Though, Hamblin does not specify what happens to the formulae related to the one that was revised. At least, resolution might serve as an instrument to show the other participant that she has a contradiction in his commitment store and ask her to 'resolve' it.

If we compare these two systems: the Obligation game and the system of formal dialectic we would see that there is a number of similarities 
and differences between them. Among the things that look similar between those systems we should mention the following:

- Both Obligation game and the formal dialectic are standard twovalued systems. That might explain why Hamblin does not provide us with any special semantical model for them.

- Both systems have two leveled syntax: (1) syntax of the object language and inference rules (that are not explicitly specified though) and (2) structural rules ${ }^{10}$. Structural rules determine the protocol of communication between participants (or agents): in the case of Obligation game between opponent and respondent, and between the opponent and proponent, or Black and White, in the case of dialectic system.

- Both systems have a notion of commitment store which is dynamic, i.e., a set of propsitional commitments that alter depending upon the moves the players make in the dialogue ${ }^{11}[26$, p. 35].

As concerns the differences between the Obligation game and formal dialectic, we would like to mark out the following:

- The organization of commitment stores is different. In case of the Obligation game there is only one commitment store, that of the respondent, because we should trace only his responses. And it is connected to the second difference.

- The roles of agents in the Obligation game are asymmetric as it was specified earlier whereas in the dialectic system they are symmetrical except for the fact that White moves first.

\footnotetext{
${ }^{10}$ It is worth mentioning that the logical and the structural rules in the dialogue logic by P. Lorenzen and K. Lorenz [17] share the same idea of two levels of rules.

${ }^{11}$ We might also intoduce a notion of static commitment store that is not altered by moves in the dialogue and it's contents are fixed before the dialogue commences [26, $\mathrm{p}$. 35]. That notion of commitment store would reflect the idea of common knowledge set $K_{C}$, especially if we take the interpretation according to which the Respondent may deny irrelevant propositions following from $K_{C}$ in order not to lose.
} 
- Another difference concerning commitment store is that in the case of Obligation game nothing can be deleted from it. That means that no retraction is possible, whereas in case of formal dialectic it is possible and players are able not only to insert commitments but also delete them by retracting their previous propositions. The latter brings about a problem for interpretation of the system.

- In relation to the commitment store, there is also difference in the ending of the game, because, as for the Obligation game, Ch. Hamblin clearly specifies the end of the game and the winning-rule whereas in case of formal dialectic he does neither the first, nor the second. That means that the game is potentially endless (if it even may be considered as a game) and there are no winning rules. Thus, we suppose that Obligation game and formal dialogue are two materially different types of dialogues: the former represents the antagonistic type, and the later is non-antagonistic. We also consider the obligation game to be a restricted type of the formal dialectic there two players are identical with respect to their knowledge and inference capacity. the difference between them is functional.

- In the case of Obligation game one of the players is forced to make certain moves (or at least we can interpret the correctness rule in that spirit), but as for the formal dialectic the system is semantically open, which means that "there is no statement at all, even a tautological one, which a speaker can unconditionally be forced to utter, nor any set of statements of which he can be unconditionally forced to utter one" [9, p. 259].

\section{Why Obligation Games are not Disputations de Obligationibus?}

In the previous sections (2 and 3 ) we have specified two possible formal representations of the medieval disputations de obligationibus. The question that arises is whether the Obligation game is actually a formalization of disputations de obligationibus or just a way to illustrate an example of a formal dialectic dialogue inspired by those disputations. In 
this section, we shall try to show that Obligation games are not adequate representations of disputations de obligationibus.

We insist that there are some crucial differences between $K_{C}$ and $C_{n}{ }^{12}$.

1. The first difference can be called conceptual. $K_{C}$ is interpreted as a common knowledge set, that should be shared by all participants of the disputation. Although we can adopt the idea that those disputations were merely a 'convenient fiction' expressed in [11], nevertheless from the conceptual point of view $K_{C}$ represents a set of agent's knowledge and beliefs (in some formalizations even an ordered one) whereas $C_{n}$ might contain both common knowledge and beliefs ${ }^{13}$ and propositions accepted in the flow of the game. But that means that the commitment store does not distinguish between common knowledge and the propositions accepted in the game, so we can not define any priority with respect to them as is done by Lagerlund and Olsson [11].

2. The Obligation game ignores very significant notions of relevance and irrelevance. Thus, players are not forced to accept or deny propositions on the basis of their relevance to those that were accepted earlier. Though, there is a correctness rule with respect to the commitment store, nevertheless, it is not specified what happens in a game if the respondent answers incorrectly. Thus the notion of commitment store can compensate for the absence of relevance relation but only partly ${ }^{14}$.

3. There are also crucial structural changes in the disputation, i.e., Ch. Hamblin combines several steps into one that deprives the

\footnotetext{
${ }^{12}$ Here we are talking about the dynamic commitment store as it is specified for the Obligation game.

${ }^{13}$ Though Hamblin never says anything about them as if there were none or they were somehow presupposed. If we follow the former interpretation than the commitment store of the respondent is empty before the beginning of the game. However, if we adopt the latter, than the commitment store should contain all those propositions before the game starts.

${ }^{14} \mathrm{We}$ even had to use the notions of sequently relevant and sequently irrelevant propositions while considering the example of the Obligation game in section 2 .
} 
respondent of the possibility to react to each proposition independently (that leads to some dramatical changes in the ability to have a winning strategy. We shall mention below that for some games over particular propositions, which the respondent could win in the disputation de obligationibus, the opponent has a winning strategy in the Obligation game).

4. The respondent has no ability to doubt proposition (so if she does not accept a proposition, she has to accept its negation) as the Obligation game system is two-valued. On the contrary, in the disputation de obligationibus the Respondent is believed to be able to mark a proposition as doubtful. Though we do not consider this system a three-valued construction, as those doubtful propositions do not influence the reasoning (i.e. are excluded from the process of evaluation of the subsequent propositions), we still consider this as a substantial difference.

At the end of this section we would like once again to bring about the problem of winning in the medieval disputation. As C. DutilhNovaes shows [6] the respondent always has a winning strategy unless he accepts a logically contradictory positum. However, in our example presented in section 1 . the positum is not contractory. Why does the respondent lose then ${ }^{15}$ ? Where did the respondent make a mistake? This question turned out to be the most interesting one. If we carefully read the example through, we shall see that at each step the opponent offers the respondent only those propositions that follow logically either from the positum or the 'real fact' (casus). That means that all propositions accepted after the first step can be derived from it. Thus we should look for the contradiction (though, perhaps, implicit) in the first step of the game. Thus, if we pay our attention to the first round of the game, we shall see that propositum $I$ is relevant to the 'real fact' (casus $)^{16}$. By consequence, we should search for the contradiction in the set formed from the propositions put forward by the opponent in

\footnotetext{
${ }^{15}$ One can easily see that the opponent not only can win, but she even has a winning strategy in the game, so that she can force the respondent to lose.

${ }^{16} \mathrm{It}$ is even a repetition of one of the propositions of the casus, namely $b_{1}$.
} 
the casus and the positum. It is not surprising that we find an implicit contradiction there, though we lack the premise that 'something that is good by participation is not good per se' which should be a part of common knowledge $K_{C}$. We emphasize once again that we cannot reveal it by any rules of the Obligation game as there is no set $K_{C}$ in Hamblin's formal system. Thus, having combined several steps into one Hamblin has trapped the respondent into an obligation of starting a game with a contradictive set of propositions.

Finally, we should like to say a few words of the characteristics of agents represented in the Obligation game. The Obligation game does not distinguish between the common knowledge, or the old beliefs (if there were any), and those that occured after the acceptance of the positum. In that respect he respondent is less rational then in other formalizations as she cannot distinguish between the old information and the new one. There remains the question of agents' determination. On the one hand, in case of formal dialectic the respondent is free to accept or deny a proposition unless she accepts a contradiction. On the other hand, if the correctness rule has a greater power than just recommendation, that would change the situation dramaticly and make it even more determined than that of the consistency maintenance games [6] (as we have here only two possible truth-values) or other formalizations.

\section{Conclusion}

In this paper we have examined the Obligation game and Hamblin's formal dialectics in their relation to the medieval disputations de obligationibus of the Burley type and other interpretations and formalizations of the latter. We have shown in sections 2, 3 and 4 that neither the Obligation game, nor formal dialectic can serve as a fruitful and adequate formalization the medieval disputation.

To sum up, the formal dialectic is a communicative protocol for rational agents that uses other formalized systems, not necessarily logic, as structural rules. We assume that it is a sort of a cognitive game for two or more participants with the objective of establishing an ordered set of propositions represented by commitment stores. It may also be 
used to check whether some set of propositions is contradictory and to eliminate any ascertained contradiction.

As for the obligation game, it is a variety of formal dialectic aimed at modeling the medieval disputation de obligtionibus. We can consider it as a game on consistency maintenance. We mark out the following features of the obligation game:

1. Common commitment store (as opposed to different commitment stores for each player in the formal dialectic);

2. Classical propositional logic (sometimes with modal fragment) as a way to set up the truth conditions for propositions;

3. Impossibility to delay the round closure. If we compare it to the dialogue logic of P. Lorenzen, we will see that there exist some limitations of round closure for intuitionistic games, though they are not so strict;

4. Impossibility to give up previously accepted propositions.

To sum up we would like to make a conclusion that neither the Obligation game, nor the formal dialectic can be assumed an adequate formalization of the disputation de obligationibus, though they show some interesting connections between the structural rules and the sets of formulae which the respondent can have a winning strategy about. It also reveals some features of the rational agents participating in the game.

\section{References}

[1] van Benthem, J. "Logical Construction Games", in: Acta Philosophica Fennica 78, Truth and Games, essays in honour of Gabriel Sandu, ed. by T. Aho and A-V Pietarinen, 2006, pp. 123-138.

[2] Boethius, A. M. S. De Hebdomadibus [http://logicmuseum.com/authors/ boethius/dehebdomadibus.htm, accessed on 26.03.2017].

[3] Brown, M.A. "The role of the Tractatus de Obligationibus in medieval logic", Franciscan Studies, 1966, Vol. 26, pp. 26-35. 
[4] Burley, W. "Obligations", in: N. Kretzmann and E. Stump (eds.), Logic and the Philosophy of Language. Cambridge University Press, 1988, pp. 369-412.

[5] Dragalina-Chernaya, E. G. "Granitsy logiki: Ontologicheskii povorot"[The Bounds of Logic: Ontological Turn], Filosofiya nauki [Philosophy of Science], 2009, No. 14, pp. 87-99. (In Russian)

[6] Dutilh Novaes, C. "Medieval Obligationes as Logical Games of Consistency Maintenance", Synthese, 2005, Vol. 145(3), pp. 371-395.

[7] Dutilh Novaes, C. "Roger Swyneshed's obligationes: A logical game of inference recognition?", Synthese, 2006, Vol. 151, pp. 125-53.

[8] Dutilh-Novaes, C. Formalizing medieval logical theories : suppositio, consequentiae and obligationes. Dordrecht, Springer, 2007. 316 pp.

[9] Hamblin, Ch. Fallacies. London, 1970. 336 pp.

[10] Hintikka, J. and Sandu, G. "What is Logic?", in: D. Jacquette (ed.), Handbook of the Philosophy of Logic, Elsevier, Amsterdam, 2007, pp. 13-40.

[11] Lagerlund, H., Olsson, E. "Disputation and Change of Belief - Burley's Theory of Obligationes as a Theory of Belief Revision", in: Yrjonsuuri, 2001, pp. 35-62.

[12] Lisanyuk, E. N. "Polemika i srednevekovyi logicheskii 'disput"' [Polemics and Medieval Logical "Disputation"], in: Kul'tura polemiki i argumentatsii ot pozdnei antichnosti do Novogo Vremeni [Culture of Polemics and Argumentation from the Late Antiquity to Modern Times], ed. by Yu. I. Ivanova. Moscow: NRU HSE, 2012, pp. 128-156. (In Russian)

[13] Lisanyuk, E. N. "Ritorika i formal'naya dialektika", [Rhetoric and Formal Dialectic], RATsIO.ru, 2010, No. 3, pp. 26-42. (In Russian)

[14] Lisanyuk, E. N. "Srednevekovyi disput", [Medieval Dispute], Logikofilosofskie shtudii [Logical and Philosophical Studies], 2006, Vyp. 4, pp. 212-228. (In Russian)

[15] Lisanyuk, E. N., Pavlova, A. M. "Logicheskie aspekty mnogoobraziya agentov", [Logical Aspects of the Diversity of Agents in Practical Reasoning], Izvetiya ural'skogo universiteta. Seriya 3. Obshchestvennye nauki [IZVESTIA Ural Federal University Journal. Series 3. Social and Political Sciences], 2016, Vol. 11, No. 4, pp. 45-60. (In Russian)

[16] Liu, F. "Diversity of agents and their Interaction", Journal of Logic, Language and information, Springer Netherlands, 2009, Vol. 18, issue 1, pp. 23-53. 
[17] Lorenzen, P., Lorenz, K. Dialogische Logik. Darmstadt, 1978. 238 pp.

[18] Rahman, Sh. "Remarks on Dialogical Meaning: A Case Study", proceedings of Proofs and Dialogues, 2011, [http://ls.informatik.unituebingen.de/prodi/slides/Rahman.pdf, accessed on 26.03.2017].

[19] Rahman, Sh., Ruckert, H. "Preface", Synthese, 2001, No. 127, pp. 1-6.

[20] Reed, Ch., Walton, D. "Argumentation Schemes in Dialogue", in: H.V. Hansen, et. al. (eds.), Dissensus and the Search for Common Ground, CD-ROM, Windsor, ON: OSSA, 2007, pp. 1-11.

[21] Read, St. "Obligations, Sophisms and Insolubles", Working paper WP6/2013/01 / St. Read, National Research University "Higher School of Economics", Moscow: Publ. House of the Higher School of Economics, 2013. 32 pp.

[22] Spade, P.V. "Three theories of obligationes: Burley, Kilvington and Swyneshed on counterfactual reasoning", History and Philosophy of Logic, Vol. 3, 1982, pp. 1-32.

[23] Uckelman, S. L. "Obligationes as Formal Dialogue Systems", in: Proceedings of the fifth starting AI researchers' symposium. Amsterdam, 2011, pp. 341-353.

[24] Uckelman, S. L., Johnston, Sp. "A Simple Semantics for Aristotelian Apodeictic Syllogistics", Advances in Modal Logic, 2010, Vol. 8, pp. 454469.

[25] Weingartner, P. Basic Questions on Truth, Kluwer Academic Publishers, 2000. 230 pp.

[26] Wells, S., Reed, Ch. "Formal Dialectic Specification", in: Argumentation in Multi-Agent Systems First International Workshop, 2004, pp. 31-43.

[27] Yrjönsuuri, M. "The role of casus in some fourteenth-century treatises on sophismata and obligations", in: K. Jacobi, ed., Argumentations theorie. Brill, Leiden, 1993, pp. 301-21.

[28] Yrjönsuuri, M. "Obligationes: 14th Century Logic of Disputational Duties", in: Acta Philosophica Fennica 55, Societas Philosophica Fennica. Helsinki, 1994. 182 pp.

[29] Yrjönsuuri, M. "Obligations as Thought Experiments", in: I. Angelelli, M. Cerezo (eds.) Studies on the History of Logic, Walter de Gruyter, Berlin, 1996, pp. 79-96.

[30] Yrjönsuuri, M. (ed.) Medieval Formal Logic. Kluwer, Dordrecht, 2001. $242 \mathrm{pp}$. 